\title{
Highly efficient and broad electromagnetic wave absorbers tuned via topology-controllable metal- organic frameworks
}

\author{
Peng Miao, Jianxin Chen, Yusheng Tang, Kai-Jie Chen and Jie Kong*
}

\begin{abstract}
Advanced electromagnetic (EM) wave absorbing materials with strong absorption and broad bandwidth are important for military stealth and elimination of microwave pollution in consumers' electronics. Metal organic framework (MOF)-derived metal/carbon hybrids with ordered structure are significantly urgent in this field. In this contribution, we presented a design strategy of hollow cage-like or solid boxlike magnetic/dielectric $\mathrm{Fe} / \mathrm{Co} / \mathrm{C}$ and dielectric $\mathrm{Fe} / \mathrm{Mn} / \mathrm{C} \mathrm{EM}$ wave absorbing nanomaterials via pyrolyzing Prussian blue's analogs with controllable topology and phase composition. The solid box-like $\mathrm{Fe} / \mathrm{Co} / \mathrm{C}$ and hollow cage-like $\mathrm{Fe} / \mathrm{Mn} / \mathrm{C}$ showed favorable absorption property with a broad effective absorption bandwidth (EAB) and a low reflection loss (RL). Especially, the EAB of $8.8 \mathrm{GHz}$ at a thickness of $2.5 \mathrm{~mm}$ for solid box-like $\mathrm{Fe} / \mathrm{Co} / \mathrm{C}$ nanocomplex prepared at $900^{\circ} \mathrm{C}$ is a new record for this type of materials. The design and tuning strategy for EM wave absorbers derived from topology-controllable MOF is important for EM functional materials possessing great potential in military stealth and consumers' electronics.
\end{abstract}

Keywords: electromagnetic wave absorber, MOF, Prussian blue's analogs, topology, pyrolysis

\section{INTRODUCTION}

Advanced electromagnetic (EM) wave absorbing materials with strong absorption, broad bandwidth, thin thickness and low density have received numerous attentions due to urgent demand in military stealth and elimination of microwave pollution or radiation in consumers' electronics and base stations of wireless networks [1-3]. Various magnetic materials, such as ferrites, $\mathrm{Fe}-, \mathrm{Co}-, \mathrm{Ni}-$ based nanoparticles and alloys, as well as carbon family materials including carbon fibers, carbon nanotubes and graphenes were mainly utilized to match the engineering applications of EM wave absorbing materials in form of coating, sealing or composites $[4,5]$.

Metal-organic frameworks (MOFs) integrated the features of organic-inorganic hybrids, high porosity, ultrahigh specific surface area and tunable topological structure [6,7]. Their diversity of compositions and topological structures give favorable functionalities with great potential in gas separation/storage, catalysis and biomedicine [8-11], etc. In our previous work [6], a synergistic sorbent separation method was presented for the one-step production of polymer-grade $\mathrm{C}_{2} \mathrm{H}_{4}$ from ternary $\left(\mathrm{C}_{2} \mathrm{H}_{2} / \mathrm{C}_{2} \mathrm{H}_{6} / \mathrm{C}_{2} \mathrm{H}_{4}\right)$ or quaternary $\left(\mathrm{CO}_{2} / \mathrm{C}_{2} \mathrm{H}_{2} / \mathrm{C}_{2} \mathrm{H}_{6} / \mathrm{C}_{2} \mathrm{H}_{4}\right)$ gas mixtures by using MOFs. Especially, MOF-derived metal/carbon composites after pyrolysis have drawn significant attention in various fields [12-14]. They might possess desirable EM wave absorbing or shielding ability. As expected, the derived $\mathrm{Co} / \mathrm{C}$ composite with well-dispersed magnetic cobalt nanoparticles inherited the topological structure of ZIF-67 crystals, showing effective absorption even at high temperature, i.e., a minimum reflection loss $\left(\mathrm{RL}_{\min }\right)$ of $-35.3 \mathrm{~dB}$ and an effective $\mathrm{ab}$ sorption bandwidth (EAB) of $5.8 \mathrm{GHz}$ measured at $500^{\circ} \mathrm{C}$ [15]. The modified ZIF-67-derived hybrids with $\mathrm{SiC}$ [16], ZIF-8 [17,18], MXene [19], poly(dimethylsilylene)diacetylene (PDSDA) [20], carbon nanotubes (CNTs) [21], multiwalled CNTs (MWCNTs) [22,23], $\mathrm{V}_{2} \mathrm{O}_{3}[24], \mathrm{Co}_{9} \mathrm{~S}_{8}$ [25], etc., were extensively studied. Meanwhile, other MOFs including two-dimensional (2D) Co-MOF [26], Co-MOF-74 [27], MIL-88A(Fe) [28], MIL-101(Fe) [29], CPT-1-Co [30], and Fe ${ }^{\text {III }}$-MOF-5 [31] with controllable topologies also received attentions in the fields of EM functional materials.

Prussian blue (PB) and its analogs (PBAs) are a class of

MOE Key Laboratory of Materials Physics and Chemistry in Extraordinary Conditions, Shaanxi Key Laboratory of Macromolecular Science and Technology, School of Chemistry and Chemical Engineering, Northwestern Polytechnical University, Xi'an 710072, China

* Corresponding author (email: kongjie@nwpu.edu.cn) 
MOFs with cubic structure as well as a general chemical formula of $\mathrm{A}_{2} \mathrm{M}\left[\mathrm{M}^{\prime}(\mathrm{CN})_{6}\right]$ ( $\mathrm{A}=$ alkaline metal ions, zeolitic water, $\mathrm{M} / \mathrm{M}^{\prime}=$ transition metals) $[32,33]$. The octahedral $\left[\mathrm{M}(\mathrm{CN})_{6}\right]^{n-}$ complex is coordinating $\mathrm{M}^{n+}$ with nitrogen bonding. Their crystal structures were verified by Keggin and Miles [34]. Based on PBAs, a series of complexes were synthesized with hollow, core shell or solid topologies showing potentials in electrocatalysts [35], energy storage [36], electrocatalytic water splitting [37], and EM wave absorbing materials [38]. For example, the $\mathrm{Fe} / \mathrm{C}$ nanocubes derived from $\mathrm{PB}$ show a $\mathrm{RL}_{\min }$ of $-22.6 \mathrm{~dB}$ at $15 \mathrm{GHz}$ and an $\mathrm{EAB}$ of $5.3 \mathrm{GHz}(12.7-$ $18 \mathrm{GHz}$ ) with a thickness of $2 \mathrm{~mm}$ [39]. The $\mathrm{Fe} / \mathrm{Co} / \mathrm{C}$ complex can also be obtained through the pyrolysis of PBAs, whose absorption is superior to that of single metallic complex [40].

As well-known, besides the suitable EM parameters to ensure impedance matching and energy attenuation [41], the topological structure of MOF derivates can also direct EM wave toward multi scattering and polarization loss $[38,42]$. These factors can be combined to enhance microwave attenuation and broaden EAB. Actually, some complicated factors, such as phase composition, morphology and temperature, affect microwave attenuation [43]. But the previous design mainly focused on the EM parameters of a specific absorber in a given condition of morphology or phase composition. However, the comprehensive effects of topology and phase composition were seldom considered. So EM wave absorption efficiency, either EAB or RL, was not favorable as expected.

In this contribution, we presented a design strategy of hollow cage-like or solid box-like magnetic/dielectric Fe/ $\mathrm{Co} / \mathrm{C}$ and dielectric $\mathrm{Fe} / \mathrm{Mn} / \mathrm{C}$ EM wave absorbing nanocomplexes via pyrolyzing PBAs with controllable topology and phase composition. The hollow cage- or solid box-like $\mathrm{Fe} / \mathrm{Co} / \mathrm{C}$ and hollow $\mathrm{Fe} / \mathrm{Mn} / \mathrm{C}$ nanocomplexes show a broad and favorable absorbing ability from the viewpoint of either $\mathrm{EAB}$ or RL. Especially, the $\mathrm{EAB}$ of $8.8 \mathrm{GHz}$ at a thickness of $2.5 \mathrm{~mm}$ for solid box-like $\mathrm{Fe} /$ $\mathrm{Co} / \mathrm{C}$ nanocomplex prepared at $900^{\circ} \mathrm{C}$ is a new record for this type of materials. It is definitely important for the design of highly efficient EM wave absorbing materials in a comprehensive perspective, which possess great potential in antenna housings, areo-engines and stealth aircrafts.

\section{EXPERIMENTAL SECTION}

\section{Synthesis of hollow Fe-Co PBA cages}

The hollow Fe-Co PBA cages were synthesized by che- mical co-precipitation as reported previously [44]. Briefly, a solution A was formed by dissolving $0.2 \mathrm{~g}$ of potassium hexacyanoferrate (III) in $60 \mathrm{~mL}$ of deionized water. Cobalt (II) acetate tetrahydrate $(0.3 \mathrm{~g})$, trisodium citrate dihydrate $(0.3 \mathrm{~g})$ and polyvinyl pyrrolidone (PVP, $3.0 \mathrm{~g}$, $\mathrm{K} 30, M_{\mathrm{w}}=40,000 \mathrm{Da}$ ) were dissolved in $40 \mathrm{~mL}$ of deionized water to form a solution B. Solution B was slowly added to solution A within 1 min under magnetic stirring. After being stirred continuously for $1 \mathrm{~min}$, the mixed solution was transferred to an oven and reacted at $80^{\circ} \mathrm{C}$ for $24 \mathrm{~h}$. The purple-black precipitation was washed with deionized water and ethanol for three times, and dried overnight at $70^{\circ} \mathrm{C}$.

\section{Synthesis of hollow Fe-Mn PBA cages}

Similarly, the hollow Mn-Fe PBA cages were obtained through a method modified from Ref. [45]. Typically, $16.4 \mathrm{mg}$ of $\mathrm{K}_{3}\left[\mathrm{Fe}(\mathrm{CN})_{6}\right]$ was dissolved in a $\mathrm{C}_{2} \mathrm{H}_{5} \mathrm{OH} / \mathrm{H}_{2} \mathrm{O}$ $(5 / 5 \mathrm{~mL})$ system under agitated stirring to give a transparent solution C. Solution D was prepared by dissolving $25.4 \mathrm{mg} \mathrm{MnSO}_{4} \cdot \mathrm{H}_{2} \mathrm{O}, 15.0 \mathrm{mg}$ trisodium citrate dihydrate and $0.3 \mathrm{~g}$ PVP $(\mathrm{K}-30)$ in a $\mathrm{C}_{2} \mathrm{H}_{5} \mathrm{OH} / \mathrm{H}_{2} \mathrm{O}(5 / 5 \mathrm{~mL})$ system under stirring for $30 \mathrm{~min}$. Solution $\mathrm{C}$ was added to solution D slowly with a syringe $(20 \mathrm{~mL})$. After being stirred for $10 \mathrm{~min}$ at room temperature, the mixed solution aged for $24 \mathrm{~h}$ without interruption. The white precipitate was collected by filtration and washed with ethanol for three times, and the hollow Fe-Mn PBA cages were obtained by drying the sample in an oven at $70^{\circ} \mathrm{C}$.

\section{Synthesis of solid Fe-Co PBA box}

The solid Fe-Co PBA boxes were synthesized by the chemical co-precipitation method as reported previously [46], which is similar to the synthesis of hollow Fe-Co PBA cages, except for the addition of trisodium citrate dihydrate and PVP in the solution B.

\section{Synthesis of solid Fe-Mn PBA box}

The solid Mn-Fe PBA boxes were synthesized by the coprecipitation method [45], similar to the synthesis of hollow Fe-Mn PBA cages, except for the addition of trisodium citrate dihydrate in the solution $\mathrm{D}$. The resultant white precipitates were collected by centrifugation, washed with ethanol for several times, and finally dried in an oven at $70^{\circ} \mathrm{C}$ overnight.

\section{Preparation of $\mathrm{Fe} / \mathrm{Co} / \mathrm{C}$ and $\mathrm{Fe} / \mathrm{Mn} / \mathrm{C}$ nanocomplexes}

The as-synthesized hollow Fe-Co or Fe-Mn PBA cages and solid Fe-Co or Fe-Mn PBA boxes were pyrolyzed in a tube-furnace (GSL-1700X, Kejing New Mater. Ltd., Hefei, 
China) at 800 and $900^{\circ} \mathrm{C}$, respectively, for $2 \mathrm{~h}$ with a heating rate of $0.5 \mathrm{~K} \mathrm{~min}^{-1}$ in argon. The nanocomplexes were named as hollow $\mathrm{Fe} / \mathrm{Co} / \mathrm{C}-800$, hollow $\mathrm{Fe} / \mathrm{Co} / \mathrm{C}-900$, solid $\mathrm{Fe} / \mathrm{Co} / \mathrm{C}-800$, solid Fe/Co/C-900, hollow Fe/Mn/C800, hollow Fe/Mn/C-900, solid Fe/Mn/C-800 and solid $\mathrm{Fe} / \mathrm{Mn} / \mathrm{C}-900$ according to topological structures and pyrolyzed temperatures, respectively.

\section{Characterizations}

$\mathrm{X}$-ray diffraction (XRD) patterns of the samples were collected on a Shimadzu $7000 \mathrm{XRD}$ using $\mathrm{Cu} \mathrm{Ka}$ radiation $(\lambda=0.15406 \mathrm{~nm})$ with a scanning voltage of $30 \mathrm{kV}$, and a scanning current of $30 \mathrm{~mA}$. The morphology and structure were scanned using a field emission scanning electron microscope (FESEM) (FEI, Verios G4) and transmission electron microscope (TEM) (FEI, Talos F200X) equipped with energy dispersive spectroscopy (EDS). Element composition was analyzed by X-ray photoelectron spectroscopy (XPS) on a Kratos Axis Ultra DLD using $\mathrm{Al} \mathrm{Ka}$ radiation $(h v=1486.6 \mathrm{eV})$ with a scanning voltage of $15 \mathrm{kV}$, and a scanning current of $10 \mathrm{~mA}$. The magnetic performance was surveyed by hysteresis loop using vibrating sample magnetometers (Lake Shore VSM 7307). The Brunauer-Emmet-Teller (BET) specific surface area and pore size distribution were measured by high-performance multi-channel fully automatic surface and pore analyzer (BeiSbiDe3H2000PS2, China) using $\mathrm{N}_{2}$ at $77 \mathrm{~K}$. Thermogravimetric analysis (TGA) (NETZSCH STA449, Germany) was car- ried out under a flow of argon with a temperature ramp of $10^{\circ} \mathrm{C} \mathrm{min}{ }^{-1}$ from room temperature to $1000^{\circ} \mathrm{C}$.

\section{EM wave absorption measurement}

The EM parameters of the nanocomplexes were measured by a vector network analyzer (VNA, Anritsu, MS46322B) using the transmission/reflection coaxial method. The composite (absorbers/paraffin $=1: 2$, wt.\%) was pressed into a coaxial ring with an outer diameter $\left(\phi_{\mathrm{o}}\right)$ of $7.00 \mathrm{~mm}$ and an inner diameter $\left(\phi_{\mathrm{i}}\right)$ of $3.04 \mathrm{~mm}$ in the frequency range of $2-18 \mathrm{GHz}$.

\section{RESULTS AND DISCUSSION}

\section{PBAs and derived $\mathrm{Fe} / \mathrm{Co} / \mathrm{C}$ and $\mathrm{Fe} / \mathrm{Mn} / \mathrm{C}$ nanocomplexes with controllable topology}

The synthetic routes of PBAs and their pyrolyzed nanocomplexes are schematically illustrated in Fig. 1. The hollow cages and solid boxes of Fe-Co PBAs or Fe-Mn PBAs were synthesized through coprecipitation methods, where the ion exchange reaction of bivalent $\mathrm{Co}^{2+}$ or $\mathrm{Mn}^{2+}$ ions and cyanide ferrate occurred in deionized water or ethanol/deionized water solution. The as-synthesized hollow Fe-Co PBA cages (Fig. 2a), hollow Fe-Mn PBA cages (Fig. 3a) and solid Fe-Co PBA boxes (Fig. 2d), solid Fe-Mn PBA boxes (Fig. 3d) were observed in TEM images. Either hollow cages or solid boxes show a welldefined hexahedron shape with a size in the range of 200$500 \mathrm{~nm}$. Meanwhile, the EDS elemental mapping images

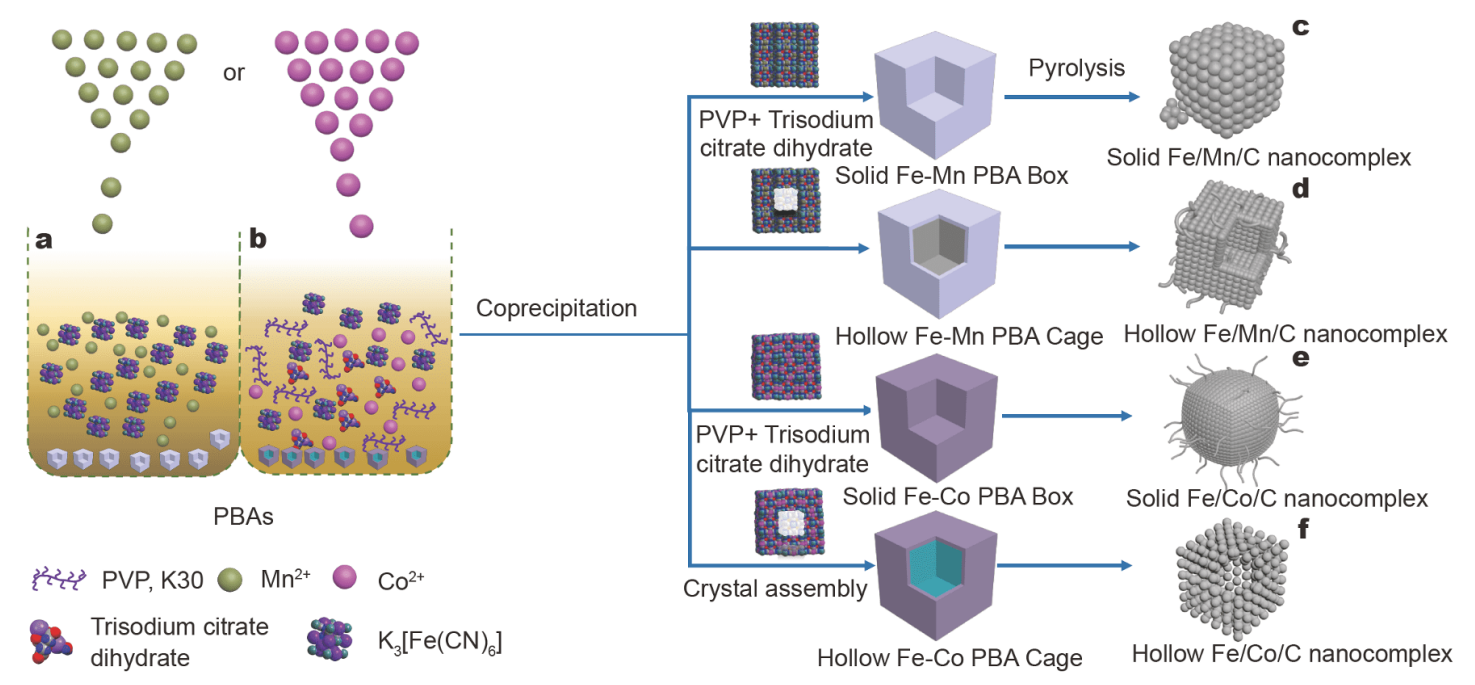

Figure 1 Schematic illustration of the fabrication route toward hollow PBA cages and solid PBA boxes and their derived nanocomplexes. (a) Synthesis of solid Fe-Co and Fe-Mn PBAs without PVP and trisodium citrate dihydrate. (b) Synthesis of hollow Fe-Co and Fe-Mn PBAs with PVP and trisodium citrate dihydrate. Preparation route of solid $\mathrm{Fe} / \mathrm{Mn} / \mathrm{C}(\mathrm{c})$, hollow $\mathrm{Fe} / \mathrm{Mn} / \mathrm{C}$ (d), solid $\mathrm{Fe} / \mathrm{Co} / \mathrm{C}$ (e) and hollow $\mathrm{Fe} / \mathrm{Co} / \mathrm{C}$ nanocomplexes (f), respectively. 

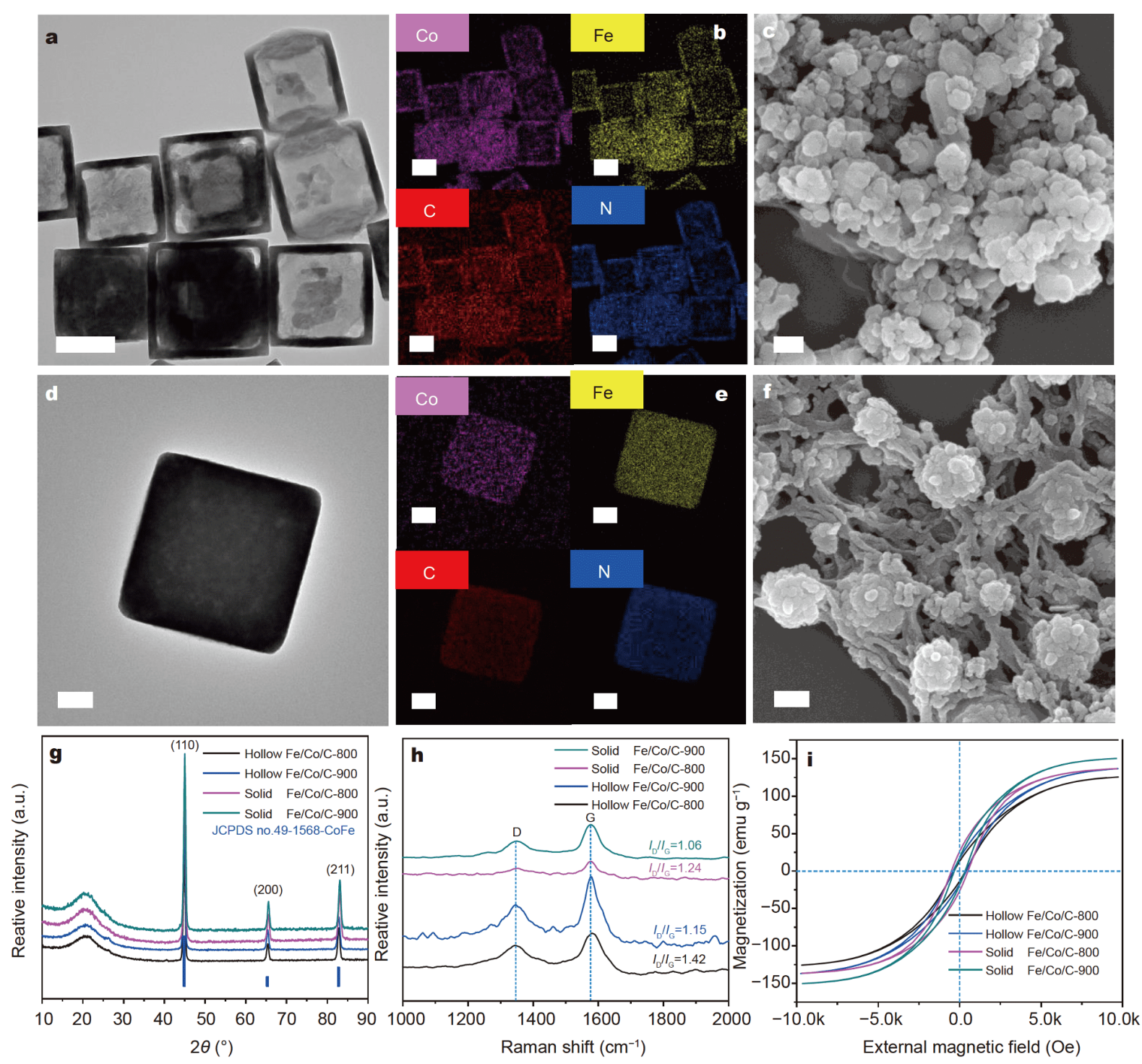

Figure 2 The topologies and phase compositions of hollow cages and solid boxes of $\mathrm{Fe}$-Co PBAs and their pyrolyzed $\mathrm{Fe} / \mathrm{Co} / \mathrm{C}$ nanocomplexes. (a) TEM image and (b) corresponding element mapping (Fe, Co, C, and N) of Fe-Co PBA hollow cages. (c) SEM image of hollow Fe/Co/C-800. (d) TEM image and (e) corresponding element mapping (Fe, Co, C, and N) of Fe-Co PBA solid boxes. (f) SEM image of solid Fe/Co/C-800. (g) PXRD patterns, (h) Raman spectra and (i) hysteresis loops of pyrolyzed $\mathrm{Fe} / \mathrm{Co} / \mathrm{C}$ nanocomplexes measured at room temperature. Scale bar: $200 \mathrm{~nm}$.

in Figs $2 b$, e and $3 b$, e clearly confirm the uniform distribution of $\mathrm{Fe} / \mathrm{Co}$ or $\mathrm{Fe} / \mathrm{Mn}$ elements in either hollow cage or solid box. The powder XRD (PXRD) patterns of hollow and solid PBAs in Fig. S1 (Supplementary information) well matched the peaks of potassium ferricyanide frameworks. The phase purity of the cubic PBAs verified their high crystallinity as supposed. The different topological structures can be obtained by controlling the usage or absence of PVP and trisodium citrate dihydrate. PVP was the key factor for forming uniform solid cubic PBAs without trisodium citrate dihydrate. The amide group of PVP would weakly coordinate with $\mathrm{Co}^{2+}$ or $\mathrm{Mn}^{2+}$ ions during the nucleation and growth of hollow $\mathrm{Fe}-\mathrm{Co}$ and $\mathrm{Fe}-\mathrm{Mn}$ PBAs. Therefore, the hollow structures of PBAs can be synthesized during the etching of trisodium citrate dihydrate $[44,47]$.

To investigate their thermal behaviors, TGA was carried out under argon atmosphere in a temperature range of $40-1000^{\circ} \mathrm{C}$. As shown in Fig. S2, the PBAs with different topologies showed similar thermolysis profiles. The initial weight loss of solid and hollow samples up to $\sim 155$ and $\sim 180^{\circ} \mathrm{C}$ can be mainly attributed to the evaporation 

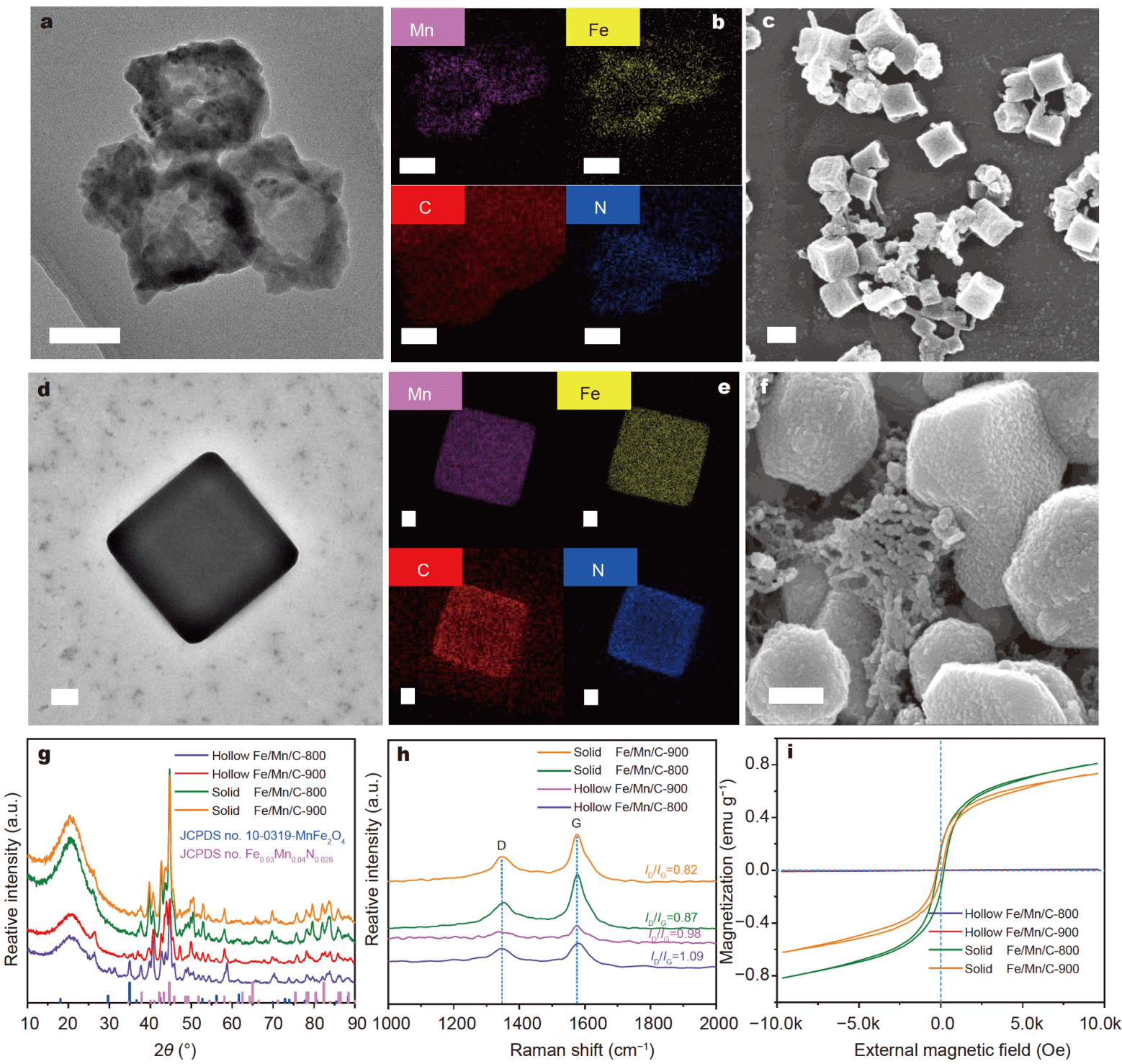

Figure 3 The topologies and phase compositions of hollow cages and solid boxes of Fe-Mn PBAs and their pyrolyzed Fe/Mn/C nanocomplexes. (a) TEM image and (b) corresponding element mapping ( $\mathrm{Fe}, \mathrm{Mn}, \mathrm{C}$, and $\mathrm{N}$ ) of Fe-Mn PBA hollow cages. (c) SEM image of hollow Fe/Mn/C-800. (d) TEM image and (e) corresponding element mapping (Fe, Mn, C, and N) of Fe-Mn PBA solid boxes. (f) SEM image of solid Fe/Mn/C-800 (g) PXRD patterns, (h) Raman spectra and (i) hysteresis loops of pyrolyzed Fe/Mn/C nanocomplexes measured at room temperature. Scale bar: $200 \mathrm{~nm}$.

of water in PBAs. As the temperature rose, the decomposition and weight loss of solid and hollow PBAs occurred above $550^{\circ} \mathrm{C}$. To ensure the ideal preparation of EM wave absorbing nanocomplex, the pyrolysis temperature of $800-900^{\circ} \mathrm{C}$ might be a good choice. After pyrolysis of PBAs, the obtained $\mathrm{Fe} / \mathrm{Co} / \mathrm{C}$ and $\mathrm{Fe} / \mathrm{Mn} / \mathrm{C}$ nanocomplexes were partially collapsed and nearly remained the hollow cage-like or solid box-like shapes as depicted in Fig. 1c-f. For solid Fe/Co/C (Fig. 2f) and Fe/
$\mathrm{Mn} / \mathrm{C}$ (Fig. 3f) nanocomplexes, some nanoparticles gradually dissociated from the box surfaces, where the carbon nanorods were generated via transition metal catalysis. The solid $\mathrm{Fe} / \mathrm{Co} / \mathrm{C}$ nanocomplex was wrapped with more nanorods than the solid $\mathrm{Fe} / \mathrm{Mn} / \mathrm{C}$ nanocomplex under the same pyrolysis temperature. Obviously, for the hollow $\mathrm{Fe} / \mathrm{Co} / \mathrm{C}$ nanocomplex (Fig. 2c), some nanoparticles were filled on or between cubic cages. On the contrary, the hollow cages of $\mathrm{Fe} / \mathrm{Mn} / \mathrm{C}$ nanocomplex 
(Fig. 3c) were identical only with a few collapsed particles. PXRD was utilized to confirm their phase compositions and crystalline features. As shown in Fig. $2 \mathrm{~g}$, the diffraction peaks of hollow and solid $\mathrm{Fe} / \mathrm{Co} / \mathrm{C}$ nanocomplexes at $44.9^{\circ}, 65.3^{\circ}$ and $82.7^{\circ}$ can be assigned to the (100), (200) and (211) planes of cubic CoFe crystal (JCPDS \# No. 49-1568). By contrast, as shown in Fig. 3g, the diffraction peaks of hollow and solid Fe/Mn/C nanocomplexes match well with $\mathrm{MnFeO}_{4}$ crystal (JCPDS \# No. 10-0319) and $\mathrm{Fe}_{0.93} \mathrm{Mn}_{0.04} \mathrm{~N}_{0.03}$ crystal (JCPDS \# No. 47-1048). The special Fe-Co or Fe-Mn phase was well formed in the nanocomplex after pyrolysis in argon atmosphere.

For the carbon species in the nanocomplex, the Raman spectra in Figs $2 \mathrm{~h}$ and $3 \mathrm{~h}$ show two typical peaks at $1350 \mathrm{~cm}^{-1}$ (D band) and $1580 \mathrm{~cm}^{-1}$ (G band). As well known, D band is related to defect/disordered carbons and $\mathrm{G}$ band represents stretching vibration of $\mathrm{sp}^{2}$ graphitic carbons, where the integral intensity ratio $\left(I_{\mathrm{D}} / I_{\mathrm{G}}\right)$ can be applied to evaluate the graphitization degree of carbons [20]. The $I_{\mathrm{D}} / I_{\mathrm{G}}$ for solid $\mathrm{Fe} / \mathrm{Co} / \mathrm{C}-800$, solid $\mathrm{Fe} /$ $\mathrm{Co} / \mathrm{C}-900$, hollow Fe/Co/C-800 and hollow Fe/Co/C-900 (the number denotes pyrolytic temperature) were 1.24, $1.06,1.42$ and 1.15 , respectively. Similarly, the $I_{\mathrm{D}} / I_{\mathrm{G}}$ ratios for solid $\mathrm{Fe} / \mathrm{Mn} / \mathrm{C}-800$, solid Fe/Mn/C-900, hollow $\mathrm{Fe} /$ $\mathrm{Mn} / \mathrm{C}-800$ and hollow Fe/Mn/C-900 were 0.87, 0.82, 1.09 and 0.98 , respectively. The decreased $I_{\mathrm{D}} / I_{\mathrm{G}}$ at higher temperature indicates the positive contribution for enhancing the graphitization degree of nanocomplex. Both hollow Fe/Co/C and hollow Fe/Mn/C show higher $I_{\mathrm{D}} / I_{\mathrm{G}}$ than their solid analogues at the same pyrolysis temperature. Since high $I_{\mathrm{D}} / I_{\mathrm{G}}$ means more defects or disorders that will lead to low electronic conductivity and high dielectric loss [48], the comprehensive effect of graphitization degree is very important for EM wave absorption as discussed later.

The PBAs and derived nanocomplexes with different topologies displayed typical type-IV isotherms with hysteresis loops in the relative pressure $\left(P / P_{0}\right)$ range of $0.6-$ 1.0 (Figs S3-S5). The BET specific surface area of the nanocomplexes gradually decreased as elevating pyrolytic temperature. The pore size was mainly below $32 \mathrm{~nm}$ (Table S1). During the transformation from PBAs to nanocomplexes, as shown in Figs 2 and 3, the decomposition of organic components and the collapse of cubic frameworks occurred at the same time at high temperatures. It also led to a rapid decrease in the specific surface area of the resultant nanocomplex. Anyway, the hollow topology and mesoporous structure are helpful to reduce the density and enhance the multi-RL of EM wave inside the nanocomplex.

\section{Magnetic and dielectric properties of hollow and solid Fe/ $\mathrm{Co} / \mathrm{C}$ and $\mathrm{Fe} / \mathrm{Mn} / \mathrm{C}$ nanocomplexes}

Considering that magnetic response is a crucial factor to EM wave absorption, the dependence of magnetic field on the magnetization of nanocomplexes is shown in Figs $2 \mathrm{i}$ and 3i. A significant hysteresis loop was observed. From the saturation magnetization $\left(M_{\mathrm{s}}\right)$, coercive force $\left(O_{\mathrm{e}}\right)$ and magnetic susceptibility $(\chi>0)$ shown in Table S1, it can be concluded that all the $\mathrm{Fe} / \mathrm{Co} / \mathrm{C}$ nanocomplexes display ferromagnetic behavior due to the unsaturated $3 \mathrm{~d}$ orbital electron interaction between the ferromagnetic metal iron and cobalt. On the contrary, the $M_{\mathrm{s}}, \mathrm{O}_{\mathrm{e}}$ and $\chi$ of Fe/Mn/C nanocomplexes are almost close to zero because the antiferromagnetic manganese neutralizes the ferromagnetic metallic iron in this case. Therefore, the EM wave attenuation in $\mathrm{Fe} / \mathrm{Mn} / \mathrm{C}$ nanocomplex can only be dependent on dielectric loss and multiple RL in hollow and mesoporous structure. Nevertheless, the magnetic response of either hollow or solid $\mathrm{Fe} / \mathrm{Co} / \mathrm{C}$ nanocomplexes is positive to EM wave absorption.

In general, complex permittivity $\left(\varepsilon_{\mathrm{r}}=\varepsilon^{\prime}-j \varepsilon^{\prime \prime}\right)$ and permeability $\left(\mu_{\mathrm{r}}=\mu^{\prime}-j \mu^{\prime \prime}\right)$ are often used as key EM parameters to evaluate the loss mechanism of absorbers [49]. The impedance $(\eta=\sqrt{\mu / \varepsilon})$ of EM absorption materials determines whether the incident EM wave is reflected or transmitted on the material surface. The EM parameters can be tuned by the phase composition and topology of the nanocomplexes. The $\varepsilon^{\prime}$ and $\varepsilon^{\prime \prime}$ in Fig. S6 decreased from $\sim 20$ to $\sim 3$ and from $\sim 13$ to $\sim 1$ for all the $\mathrm{Fe} / \mathrm{Co} / \mathrm{C}$ nanocomplexes, respectively. The $\varepsilon^{\prime}$ and $\varepsilon^{\prime \prime}$ of solid $\mathrm{Fe} / \mathrm{Co} /$ C-900 were higher, indicating higher energy storage and dielectric loss of EM wave. The distinct peak bump of $\varepsilon^{\prime \prime}$ in $3-8 \mathrm{GHz}$ for solid $\mathrm{Fe} / \mathrm{Co} / \mathrm{C}-800$ indicated strong dielectric loss capacity. In contrast, the $\varepsilon^{\prime}$ and $\varepsilon^{\prime \prime}$ of solid nanocomplexes were both higher than hollow ones under the same pyrolysis temperature in most cases. For Fe/Mn/ C nanocomplexes, the $\varepsilon^{\prime}$ ranging from $\sim 16$ to $\sim 4$ also exhibited dispersion behavior as the frequency increased (Fig. S7). However, the dependence of $\varepsilon^{\prime \prime}$ on frequency showed a relatively violent fluctuation. In the same way, the complex permittivity for solid $\mathrm{Fe} / \mathrm{Mn} / \mathrm{C}$ was higher than that for the hollow ones under the same pyrolytic conditions in most cases. The permittivity is related to the degree of graphitization and resultant electronic conductivity. As shown in Fig. 3, the enhanced permittivity is mainly due to some carbon nanorods existing in the solid $\mathrm{Fe} / \mathrm{Mn} / \mathrm{C}$ nanocomplex. 
Fig. S8 illustrates the frequency dependence of complex permeability for ferromagnetic hollow and solid $\mathrm{Fe} / \mathrm{Co} / \mathrm{C}$ nanocomplexes derived at different pyrolytic temperatures. The $\mu^{\prime}$ and $\mu^{\prime \prime}$ of solid $\mathrm{Fe} / \mathrm{Co} / \mathrm{C}$ are higher than that of hollow $\mathrm{Fe} / \mathrm{Co} / \mathrm{C}$ in most cases. The initial susceptibility $(x>0)$ in Table S1 indicated ferromagnetic response behavior according to the following Equations (1-3) [50]:

$\chi=M / H$,

$\mu^{\prime}=1+(M / H) \cos \sigma$,

$\mu^{\prime \prime}=1+(M / H) \sin \sigma$,

where $M$ is the magnetization, $H$ the external magnetic field, and $\sigma$ the phase lag angle of magnetization behind the external magnetic field.

For the $\mathrm{Fe} / \mathrm{Co} / \mathrm{C}$ nanocomplex, the complex permeability is distinctly different with frequency, where the $\mu^{\prime}$ varies from 0.8 to 1.6 and the $\mu^{\prime \prime}$ varies from 0 to 0.7 , respectively. The solid $\mathrm{Fe} / \mathrm{Co} / \mathrm{C}-800$ shows the highest $\mu^{\prime}$ and $\mu^{\prime \prime}$ among all the $\mathrm{Fe} / \mathrm{Co} / \mathrm{C}$ nanocomplexes. If the pyrolysis temperature rises to $900^{\circ} \mathrm{C}$, which is close to the Curie temperature of metallic cobalt, the permeability will decrease. Compared with $\mu^{\prime}$ and $\mu^{\prime \prime}$, the saturation magnetization is less than 1 for all the $\mathrm{Fe} / \mathrm{Mn} / \mathrm{C}$ nanocomplexes. It should be pointed out that the permeability for some samples in 6-18 GHz increased as presented in Figs S8 and S9. Although it is abnormal due to the Snoek limit for traditional ferromagnetic materials, the microcurrent loop and EM induction induced by heterostructure anisotropy might be the main reason [51]. As illustrated in Figs $2 \mathrm{f}$ and $3 \mathrm{f}$, the graphitic carbons or nanorods were distributed on or around the surface of cubic Fe/Mn or $\mathrm{Fe} / \mathrm{Co}$ particles to form the heterostructure. Similar to expanded graphite $/ \mathrm{Fe}_{3} \mathrm{O}_{4}$ nanoring composites [51], the conductive networks were formed with the graphitic carbons or nanorods and cubic $\mathrm{Fe} / \mathrm{Mn}$ or $\mathrm{Fe} / \mathrm{Co}$ particles in the nanocomplexes. The microcurrent loop could be formed around different interfaces of cubic magnetic nanoparticles, resulting in EM induction. It might be the main reason for the increase of permeability for either $\mathrm{Fe} /$ $\mathrm{Co} / \mathrm{C}$ or $\mathrm{Fe} / \mathrm{Mn} / \mathrm{C}$ nanocomplexes. At the same time, the magnetic hysteresis loop almost coincides with the initial response curve. The initial susceptibility $(\chi=0)$ in Table $S 1$ indicates that the contribution of magnetic loss for EM wave absorption can be neglected. The dielectric loss will play a key role for $\mathrm{Fe} / \mathrm{Mn} / \mathrm{C}$ nanocomplexes.

EM wave absorption performance of hollow and solid Fe/ $\mathrm{Co} / \mathrm{C}$ and $\mathrm{Fe} / \mathrm{Mn} / \mathrm{C}$ nanocomplexes

The EM wave RLs of hollow and solid $\mathrm{Fe} / \mathrm{Co} / \mathrm{C}$ and $\mathrm{Fe} /$
$\mathrm{Mn} / \mathrm{C}$ nanocomplexes were calculated based on the complex permittivity and permeability according to the transmission line theory using the following Equations (4) and (5) [52,53].

$\mathrm{RL}=20 \log _{10}\left|\frac{Z_{\text {in }}-1}{Z_{\text {in }}+1}\right|$,

$Z_{\text {in }}=\sqrt{\frac{\mu_{\mathrm{r}}}{\varepsilon_{\mathrm{r}}}} \tanh \left[j \frac{2 \pi f d}{c} \sqrt{\mu_{\mathrm{r}} \varepsilon_{\mathrm{r}}}\right]$,

where $Z_{\text {in }}, \mu_{\mathrm{r}}$ and $\varepsilon_{\mathrm{r}}$ are the normalized input impedance, permittivity, and permeability, respectively. The $f, d$ and $c$ represent the microwave frequency, thickness of sample and the velocity of the EM wave in vacuum, respectively. In general, the RL value of less than $-10 \mathrm{~dB}$ represents that EM wave is attenuated at least $90 \%$.

In Fig. 4, the RLs versus frequency for all samples at the same thicknesses of 2.0 and $2.5 \mathrm{~mm}$ are presented. It can be seen that the EM wave attenuation is strongly dependent on the phase compositions and topologies of the nanocomplexes. Compared with $\mathrm{Fe} / \mathrm{C}$ nanocomplex derived from $\mathrm{PB}$ under the same pyrolysis condition (Fig. S10), the minimum $\mathrm{RL}$ of solid $\mathrm{Fe} / \mathrm{Co} / \mathrm{C}-900$ reaches $-54.6 \mathrm{~dB}$ at $16.1 \mathrm{GHz}$ with a thickness of $2.0 \mathrm{~mm}$ (Fig. 4). The attenuation ability of EM wave is greatly improved after the introduction of cobalt. Meanwhile, the EAB of solid $\mathrm{Fe} / \mathrm{Co} / \mathrm{C}-900$ achieves $8.8 \mathrm{GHz}$ at a thickness of $2.5 \mathrm{~mm}$. The EAB and RL value show an obvious diversity with different thicknesses, pyrolysis temperatures and topological structures. The solid $\mathrm{Fe} / \mathrm{Co} / \mathrm{C}-800$ possesses lower $\mathrm{RL}$ and higher $\mathrm{EAB}$ than hollow $\mathrm{Fe} / \mathrm{Co} / \mathrm{C}-800$ at the same thickness. And the $\mathrm{EAB}$ of solid $\mathrm{Fe} / \mathrm{Co} / \mathrm{C}-900$ is also higher than that of hollow $\mathrm{Fe} / \mathrm{Co} / \mathrm{C}-900$ with a thickness of $2.5 \mathrm{~mm}$ (Fig. 4a, b). The difference in absorption is mainly caused by topological structure and the graphitization degree of carbon as depicted in Fig. 5a, b. Compared with hollow $\mathrm{Fe} / \mathrm{Co} / \mathrm{C}$, the solid $\mathrm{Fe} / \mathrm{Co} / \mathrm{C}$ wrapped with carbon nanorods possesses higher $I_{\mathrm{D}} / I_{\mathrm{G}}$ and degree of graphitization. The graphitization and carbon nanorods provide more efficient dielectric loss. Hence, the solid box-like $\mathrm{Fe} / \mathrm{Co} / \mathrm{C}$ shows strong $\mathrm{EM}$ wave absorption and broad bandwidth.

However, for the $\mathrm{Fe} / \mathrm{Mn} / \mathrm{C}$ nanocomplex, the $\mathrm{EAB}$ and $\mathrm{RL}$ for hollow $\mathrm{Fe} / \mathrm{Mn} / \mathrm{C}-800$ are obviously better than those of solid Fe/Mn/C-800. As shown in Fig. 4c, the EAB of hollow $\mathrm{Fe} / \mathrm{Mn} / \mathrm{C}-800$ is $6.7 \mathrm{GHz}$, higher than that of solid $\mathrm{Fe} / \mathrm{Mn} / \mathrm{C}-800(4.7 \mathrm{GHz})$ at the same thickness of $2.5 \mathrm{~mm}$. Similarly, the EAB of hollow $\mathrm{Fe} / \mathrm{Mn} / \mathrm{C}-900$ $(3.3 \mathrm{GHz})$ is also higher than that of solid $\mathrm{Fe} / \mathrm{Mn} / \mathrm{C}-900$ $(2.9 \mathrm{GHz})$ at the same thickness (Fig. $4 \mathrm{~d})$. The phenomenon is attributed to the hollow cage structure of 

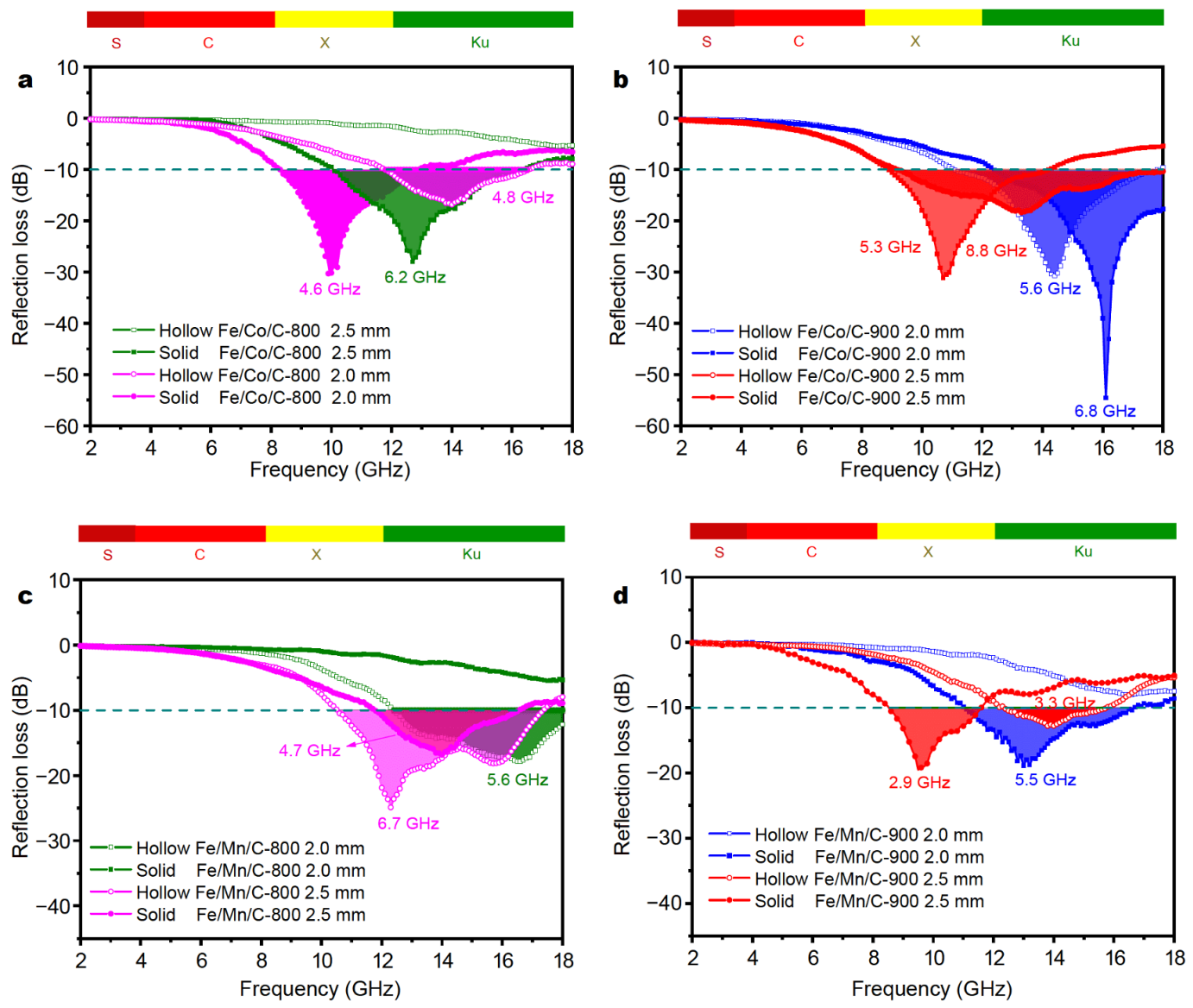

Figure 4 The comparison of EM wave absorption performance for pyrolyzed $\mathrm{Fe} / \mathrm{Co} / \mathrm{C}$ and $\mathrm{Fe} / \mathrm{Mn} / \mathrm{C}$ nanocomplexes. RL of (a) solid and hollow Fe/ Co/C-800 nanocomplexes, (b) solid and hollow Fe/Co/C-900 nanocomplexes, (c) solid and hollow Fe/Mn/C-800 nanocomplexes and (d) solid and hollow $\mathrm{Fe} / \mathrm{Mn} / \mathrm{C}-900$ nanocomplexes.

$\mathrm{Fe} / \mathrm{Mn} / \mathrm{C}$ nanocomplex. As shown in Fig. $5 \mathrm{c}$, d, the EM wave enters the hollow structure and leads to the multiple $\mathrm{RL}$ and dielectric loss, enhancing the absorption performance, i.e., high EAB and low RL. On the contrary, it is difficult for EM wave to penetrate into the interior due to the higher value of permittivity and solid structure of solid $\mathrm{Fe} / \mathrm{Mn} / \mathrm{C}-900$. In addition, the hollow $\mathrm{Fe} / \mathrm{Mn} / \mathrm{C}-800$ has broader $\mathrm{EAB}$ and $\mathrm{RL}$ than solid $\mathrm{Fe} / \mathrm{Mn} / \mathrm{C}-800$. It is confirmed that hollow structures with the suitable EM parameters can efficiently enhance the EM wave absorption.

The EM wave attenuation constant $(\alpha)$ in the interior of hollow cages and solid boxes can be accessed via the following Equation (6) [20,54]:

$$
\begin{aligned}
\alpha= & \frac{\sqrt{2} \pi \mathrm{f}}{c} \\
& \times \sqrt{\left(\mu^{\prime \prime} \varepsilon^{\prime \prime}-\mu^{\prime} \varepsilon^{\prime}\right)+\sqrt{\left(\mu^{\prime} \varepsilon^{\prime \prime}+\mu^{\prime \prime} \varepsilon^{\prime}\right)^{2}+\left(\mu^{\prime \prime} \varepsilon^{\prime \prime}-\mu^{\prime} \eta^{\prime}\right)^{2}}} .
\end{aligned}
$$

As shown in Fig. S11, an obvious increase of $\alpha$ in 8$18 \mathrm{GHz}$ can be observed for all the nanocomplexes. The solid $\mathrm{Fe} / \mathrm{Co} / \mathrm{C}-900$ exhibits the highest $\alpha$, which is consistent with the maximum absorption and $\mathrm{EAB}$ with a thickness of $2.5 \mathrm{~mm}$. Similarly, the hollow Fe/Mn/C-900 also has the maximum $\alpha$, though it does not show the best absorption bandwidth and absorption intensity as illustrated in Fig. 4d. Of course, the highest $\alpha$ cannot fully prove that the nanocomplex has strong EM wave absorption ability. It also requires impedance characteristic of absorbers close to that of the free space calculated by Equation (1). The frequency dependence of $\left|Z_{\text {in }} / Z_{0}\right|$ for all samples is shown in Figs $S 12$ and S13. The value of $\left|Z_{\text {in }}\right|$ $Z_{0} \mid$ is close to $0.8-1.2$, close to the best impedance matching. The value of $\left|Z_{\text {in }} / Z_{0}\right|$ for solid $\mathrm{Fe} / \mathrm{Co} / \mathrm{C}-900$ is close to 0.9 when the $\mathrm{EAB}$ achieves maximum at a thickness of $2.5 \mathrm{~mm}$. In this case, the incident EM wave can enter the absorber to a great extent as the optimal 

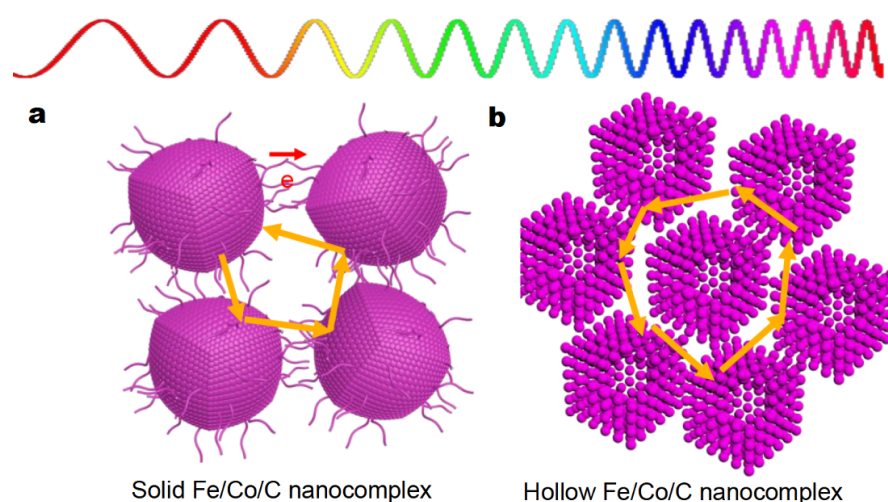

$$
\text { b }
$$
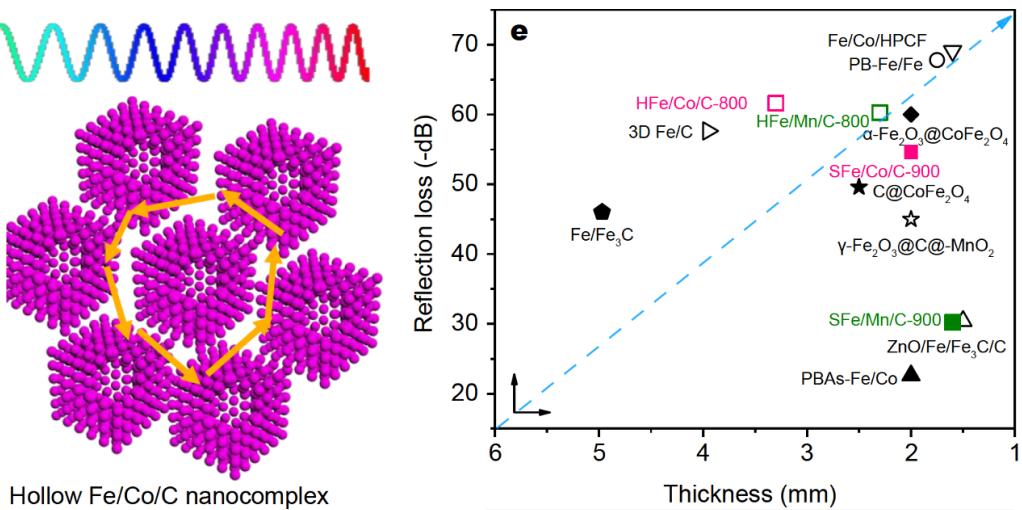

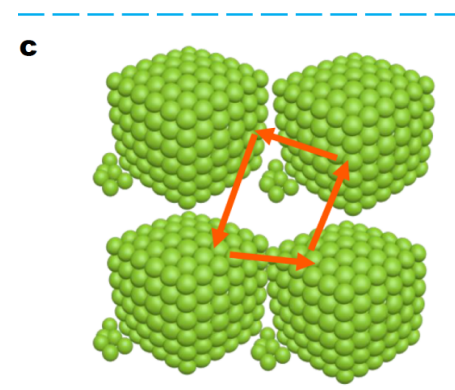

Solid Fe/Mn/C nanocomplex
Hollow $\mathrm{Fe} / \mathrm{Co} / \mathrm{C}$ nanocomplex

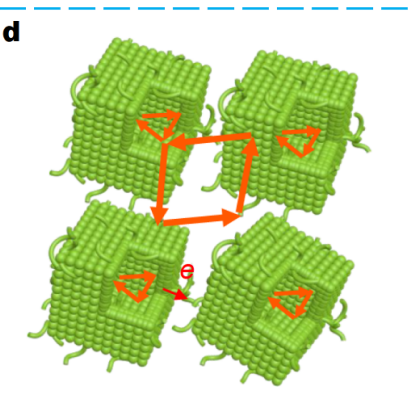

Hollow Fe/Mn/C nanocomplex

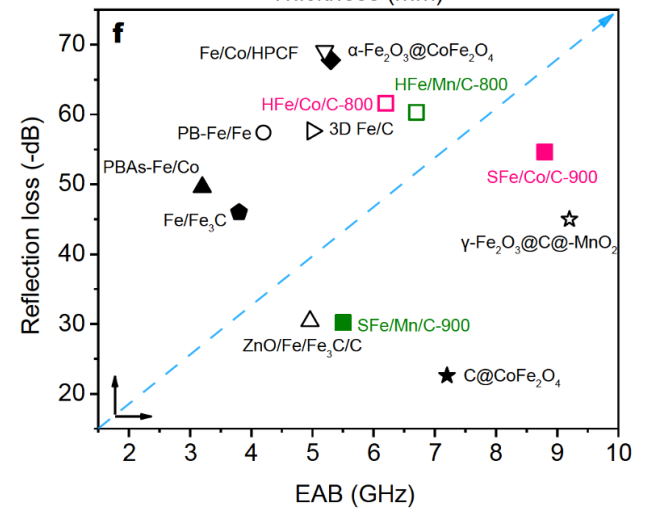

Figure 5 The proposed diagrams for multi RL and interfacial polarization mechanism of EM wave absorption. (a) Carbon network grown on the surface of solid Fe/Co/C nanocomplex, (b) the Fe/Co/C particles fall off from the surface and inside of the hollow Fe/Co/C nanocomplex, (c) the Fe/ $\mathrm{Mn} / \mathrm{C}$ particles fall off from the surface of solid $\mathrm{Fe} / \mathrm{Mn} / \mathrm{C}$ nanocomplex and (d) the carbon nanorods grown on or between hollow Fe/Mn/C nanocomplexes. The summary of EM wave absorption with (e) RL vs. thickness and (f) RL vs. EAB from different MOF-derived metal/carbon complexes.

impedance matching. However, the value of $\left|Z_{\text {in }} / Z_{0}\right|$ for solid $\mathrm{Fe} / \mathrm{Mn} / \mathrm{C}-800$ is below 0.8 , so its RL is above $-10 \mathrm{~dB}$ in correspondence with the impedance mismatching feature at a thickness of $2.0 \mathrm{~mm}$.

Compared with Fe-based hybrids or metal/carbon nanocomposites-derived MOFs reported previously, both $\mathrm{Fe} / \mathrm{Co} / \mathrm{C}$ and $\mathrm{Fe} / \mathrm{Mn} / \mathrm{C}$ nanocomplexes exhibited excellent EM wave absorption in the frequency range of 2$18 \mathrm{GHz}$. As illustrated in Fig. 5e, f and Table S2, the top right corner represents the optimal EM wave performance. The solid Fe/Co/C-900, hollow Fe/Co/C-800 and hollow $\mathrm{Fe} / \mathrm{Mn} / \mathrm{C}-800$ all show favorable optimal EM wave absorbing abilities from the viewpoint of either EAB or $\mathrm{RL}$. In details, for the $\mathrm{Fe} / \mathrm{Mn} / \mathrm{C}$ nanocomplex, the hollow $\mathrm{Fe} / \mathrm{Mn} / \mathrm{C}-800$ shows the best EM wave absorption from the viewpoint of both $\mathrm{RL}$ and $\mathrm{EAB}$. For $\mathrm{Fe} / \mathrm{Co} / \mathrm{C}$ nanocomplex, the solid $\mathrm{Fe} / \mathrm{Co} / \mathrm{C}-900$ possesses the best $\mathrm{EM}$ wave absorption. The $\mathrm{EAB}$ of $8.8 \mathrm{GHz}$ at a thickness of $2.5 \mathrm{~mm}$ for solid $\mathrm{Fe} / \mathrm{Co} / \mathrm{C}-900$ is a new record for this type of materials. As shown in Fig. 5a-d, in addition to reflection and transmission, the interface polarization is also important for absorbers. The graphitic carbons or nanorods in nanocomplexes provide more interfaces, enhancing the interface polarization. The migration of electric dipoles induced by interfacial polarization leads to dissipation of EM wave in absorbers. Therefore, a broad $\mathrm{EAB}$ was achieved in solid $\mathrm{Fe} / \mathrm{Co} / \mathrm{C}-900$ nanocomplex.

For further analysis, the EM loss mechanisms of $\mathrm{Fe} / \mathrm{Co} /$ $\mathrm{C}$ and $\mathrm{Fe} / \mathrm{Mn} / \mathrm{C}$ are different. For $\mathrm{Fe} / \mathrm{Co} / \mathrm{C}$, the magnetic loss, dielectric loss and interface polarization, as well as the carbon nanorods-wrapped FeCo magnetic core are beneficial for enhancing the EM wave absorption. The interface polarization and magnetic loss induce high EM wave attenuation. That is the reason that solid $\mathrm{Fe} / \mathrm{Co} / \mathrm{C}$ possesses better absorption than hollow $\mathrm{Fe} / \mathrm{Co} / \mathrm{C}$. On the contrary, the contribution of magnetic loss is almost zero as for $\mathrm{Fe} / \mathrm{Mn} / \mathrm{C}$, and the dielectric loss and multi reflection in the hollow cage-like nanostructure become the main factor to EM wave attenuation. In the future design 
of highly efficient EM wave absorbing materials using MOFs, the magnetic loss, dielectric loss, phase composition and topology should be considered in a comprehensive perspective.

\section{CONCLUSIONS}

The PBA hollow cages and solid boxes were synthesized through the coprecipitation method and the pyrolyzed hollow cage-like or solid box-like magnetic/dielectric $\mathrm{Fe} / \mathrm{Co} / \mathrm{C}$ and dielectric $\mathrm{Fe} / \mathrm{Mn} / \mathrm{C}$ nanocomplexes were obtained with controllable topological structures. The solid box-like $\mathrm{Fe} / \mathrm{Co} / \mathrm{C}-900$ and hollow $\mathrm{Fe} / \mathrm{Mn} / \mathrm{C}$ all showed favorable absorbing abilities from the viewpoint of either EAB or RL. For solid box-like Fe/Co/C-900, the $\mathrm{EAB}$ of $8.8 \mathrm{GHz}$ at a thickness of $2.5 \mathrm{~mm}$ is a new record for this type of materials. The tuning of EM wave absorbing ability via topology-controllable MOFs is very important for the design of absorbers. And the highly efficient and broad absorbing nanocomplexes possess great potential in antenna housings, radomes, aero-engines and stealth aircrafts.

Received 11 March 2020; accepted 5 April 2020;

published online 12 June 2020

1 Li X, Wang L, You W, et al. Morphology-controlled synthesis and excellent microwave absorption performance of $\mathrm{ZnCo}_{2} \mathrm{O}_{4}$ nanostructures via a self-assembly process of flake units. Nanoscale, 2019, 11: 2694-2702

2 Lv H, Yang Z, Wang PL, et al. A voltage-boosting strategy enabling a low-frequency, flexible electromagnetic wave absorption device. Adv Mater, 2018, 30: 1706343

3 Cao MS, Cai YZ, He P, et al. 2D MXenes: Electromagnetic property for microwave absorption and electromagnetic interference shielding. Chem Eng J, 2019, 359: 1265-1302

4 Liang C, Qin W, Wang Z. Cobalt doping-induced strong electromagnetic wave absorption in $\mathrm{SiC}$ nanowires. J Alloys Compd, 2019, 781: 93-100

5 Liu $\mathrm{L}, \mathrm{He} \mathrm{N}, \mathrm{Wu} \mathrm{T}$, et al. $\mathrm{Co} / \mathrm{C} / \mathrm{Fe} / \mathrm{C}$ hierarchical flowers with strawberry-like surface as surface plasmon for enhanced permittivity, permeability, and microwave absorption properties. Chem Eng J, 2019, 355: 103-108

6 Chen KJ, Madden DG, Mukherjee S, et al. Synergistic sorbent separation for one-step ethylene purification from a four-component mixture. Science, 2019, 366: 241-246

7 Yang QY, Lama P, Sen S, et al. Reversible switching between highly porous and nonporous phases of an interpenetrated diamondoid coordination network that exhibits gate-opening at methane storage pressures. Angew Chem Int Ed, 2018, 57: 5684-5689

8 Chen KJ, Scott HS, Madden DG, et al. Benchmark $\mathrm{C}_{2} \mathrm{H}_{2} / \mathrm{CO}_{2}$ and $\mathrm{CO}_{2} / \mathrm{C}_{2} \mathrm{H}_{2}$ separation by two closely related hybrid ultramicroporous materials. Chem, 2016, 1: 753-765

9 Manna K, Zhang T, Carboni M, et al. Salicylaldimine-based metalorganic framework enabling highly active olefin hydrogenation with iron and cobalt catalysts. J Am Chem Soc, 2014, 136: 1318213185
10 Zhang L, Wu HB, Madhavi S, et al. Formation of $\mathrm{Fe}_{2} \mathrm{O}_{3}$ microboxes with hierarchical shell structures from metal-organic frameworks and their lithium storage properties. J Am Chem Soc, 2012, 134: 17388-17391

11 He Z, Dai Y, Li X, et al. Hybrid nanomedicine fabricated from photosensitizer-terminated metal-organic framework nanoparticles for photodynamic therapy and hypoxia-activated cascade chemotherapy. Small, 2019, 15: 1804131

12 Begum S, Hassan Z, Bräse S, et al. Metal-organic frameworktemplated biomaterials: Recent progress in synthesis, functionalization, and applications. Acc Chem Res, 2019, 52: 1598-1610

13 Cao C, Ma D, Xu Q, et al. Semisacrificial template growth of selfsupporting MOF nanocomposite electrode for efficient electrocatalytic water oxidation. Adv Funct Mater, 2019, 29: 18074181807426

14 Liu Y, Yang Y, Sun Y, et al. Ostwald ripening-mediated grafting of metal-organic frameworks on a single colloidal nanocrystal to form uniform and controllable MXF. J Am Chem Soc, 2019, 141: 74077413

15 Lü Y, Wang $\mathrm{Y}, \mathrm{Li} \mathrm{H}$, et al. MOF-derived porous $\mathrm{Co} / \mathrm{C}$ nanocomposites with excellent electromagnetic wave absorption properties. ACS Appl Mater Interfaces, 2015, 7: 13604-13611

16 Zhang $\mathrm{K}, \mathrm{Wu} \mathrm{F}$, Xie $\mathrm{A}$, et al. In situ stringing of metal organic frameworks by $\mathrm{SiC}$ nanowires for high-performance electromagnetic radiation elimination. ACS Appl Mater Interfaces, 2017, 9: 33041-33048

17 Liao Q, He M, Zhou Y, et al. Highly cuboid-shaped heterobimetallic metal-organic frameworks derived from porous $\mathrm{Co} /$ $\mathrm{ZnO} / \mathrm{C}$ microrods with improved electromagnetic wave absorption capabilities. ACS Appl Mater Interfaces, 2018, 10: 29136-29144

18 Feng W, Wang Y, Chen J, et al. Metal organic framework-derived CoZn alloy/N-doped porous carbon nanocomposites: Tunable surface area and electromagnetic wave absorption properties. J Mater Chem C, 2018, 6: 10-18

19 Liao Q, He M, Zhou Y, et al. Rational construction of $\mathrm{Ti}_{3} \mathrm{C}_{2} \mathrm{~T}_{x} / \mathrm{Co}-$ MOF-Derived Laminated $\mathrm{Co} / \mathrm{TiO}_{2}-\mathrm{C}$ hybrids for enhanced electromagnetic wave absorption. Langmuir, 2018, 34: 15854-15863

20 Miao P, Cheng K, Li H, et al. Poly(dimethylsilylene)diacetyleneguided ZIF-based heterostructures for full Ku-band electromagnetic wave absorption. ACS Appl Mater Interfaces, 2019, 11: 17706-17713

21 Yin Y, Liu X, Wei X, et al. Porous CNTs/Co composite derived from zeolitic imidazolate framework: A lightweight, ultrathin, and highly efficient electromagnetic wave absorber. ACS Appl Mater Interfaces, 2016, 8: 34686-34698

22 Yin Y, Liu X, Wei X, et al. Magnetically aligned Co-C/MWCNTs composite derived from MWCNT-Interconnected zeolitic imidazolate frameworks for a lightweight and highly efficient electromagnetic wave absorber. ACS Appl Mater Interfaces, 2017, 9: 30850-30861

23 Shu R, Li W, Wu Y, et al. Nitrogen-doped Co-C/MWCNTs nanocomposites derived from bimetallic metal-organic frameworks for electromagnetic wave absorption in the X-band. Chem Eng J, 2019, 362: 513-524

24 Zhou $\mathrm{C}, \mathrm{Wu} \mathrm{C}$, Liu $\mathrm{D}$, et al. Metal-organic framework derived hierarchical Co/ $/ \mathrm{CV}_{2} \mathrm{O}_{3}$ hollow spheres as a thin, lightweight, and high-efficiency electromagnetic wave absorber. Chem Eur J, 2019, 25: 2234-2241

25 Yang Z, Lv H, Wu R. Rational construction of graphene oxide with MOF-derived porous NiFe@C nanocubes for high-performance 
microwave attenuation. Nano Res, 2016, 9: 3671-3682

26 Zhang Y, Zhang HB, Wu X, et al. Nanolayered cobalt@carbon hybrids derived from metal-organic frameworks for microwave absorption. ACS Appl Nano Mater, 2019, 2: 2325-2335

27 Wang $\mathrm{K}$, Chen $\mathrm{Y}$, Tian $\mathrm{R}$, et al. Porous Co-C core-shell nanocomposites derived from Co-MOF-74 with enhanced electromagnetic wave absorption performance. ACS Appl Mater Interfaces, 2018, 10: 11333-11342

28 Wu N, Xu D, Wang Z, et al. Achieving superior electromagnetic wave absorbers through the novel metal-organic frameworks derived magnetic porous carbon nanorods. Carbon, 2019, 145: 433444

29 Xiang Z, Song Y, Xiong J, et al. Enhanced electromagnetic wave absorption of nanoporous $\mathrm{Fe}_{3} \mathrm{O}_{4} @$ carbon composites derived from metal-organic frameworks. Carbon, 2019, 142: 20-31

30 Zhu BY, Miao P, Kong J, et al. Co/C composite derived from a newly constructed metal-organic framework for effective microwave absorption. Cryst Growth Des, 2019, 19: 1518-1524

31 Liu W, Liu L, Ji G, et al. Composition design and structural characterization of MOF-derived composites with controllable electromagnetic properties. ACS Sustain Chem Eng, 2017, 5: 79617971

32 Nai J, Lou XWD. Hollow structures based on Prussian blue and its analogs for electrochemical energy storage and conversion. Adv Mater, 2019, 31: 1706825

33 Deng L, Yang Z, Tan L, et al. Investigation of the Prussian blue analog $\mathrm{Co}_{3}\left[\mathrm{Co}(\mathrm{CN})_{6}\right]_{2}$ as an anode material for nonaqueous potassium-ion batteries. Adv Mater, 2018, 30: 1802510

34 Keggin JF, Miles FD. Structures and formulæ of the Prussian blues and related compounds. Nature, 1936, 137: 577-578

35 Nai J, Lu Y, Yu L, et al. Formation of Ni-Fe mixed diselenide nanocages as a superior oxygen evolution electrocatalyst. Adv Mater, 2017, 29: 1703870

36 Li Y, Hu J, Yang K, et al. Synthetic control of Prussian blue derived nano-materials for energy storage and conversion application. Mater Today Energy, 2019, 14: 100332

37 Du Y, Chen J, Li L, et al. Core-shell FeCo Prussian blue analogue/ $\mathrm{Ni}(\mathrm{OH})_{2}$ derived porous ternary transition metal phosphides connected by graphene for effectively electrocatalytic water splitting. ACS Sustain Chem Eng, 2019, 7: 13523-13531

38 Miao P, Zhou R, Chen K, et al. Tunable electromagnetic wave absorption of supramolecular isomer-derived nanocomposites with different morphology. Adv Mater Interfaces, 2020, 7: 1901820

39 Qiang R, Du Y, Zhao H, et al. Metal organic framework-derived $\mathrm{Fe} / \mathrm{C}$ nanocubes toward efficient microwave absorption. J Mater Chem A, 2015, 3: 13426-13434

40 Liu D, Qiang R, Du Y, et al. Prussian blue analogues derived magnetic FeCo alloy/carbon composites with tunable chemical composition and enhanced microwave absorption. J Colloid Interface Sci, 2018, 514: 10-20

41 Panwar R, Puthucheri S, Singh D. Experimental demonstration of novel hybrid microwave absorbing coatings using particle-sizecontrolled hard-soft ferrite. IEEE Trans Magn, 2018, 54: 1-5

42 Zhou M, Zhang X, Wei J, et al. Morphology-controlled synthesis and novel microwave absorption properties of hollow urchinlike $\alpha$ $\mathrm{MnO}_{2}$ nanostructures. J Phys Chem C, 2011, 115: 1398-1402

43 Zhao B, Zhao W, Shao G, et al. Morphology-control synthesis of a core-shell structured $\mathrm{NiCu}$ alloy with tunable electromagneticwave absorption capabilities. ACS Appl Mater Interfaces, 2015, 7: $12951-12960$
44 Nai J, Zhang J, Lou XWD. Construction of single-crystalline Prussian blue analog hollow nanostructures with tailorable topologies. Chem, 2018, 4: 1967-1982

45 Zheng F, Zhu D, Shi X, et al. Metal-organic framework-derived porous $\mathrm{Mn}_{1.8} \mathrm{Fe}_{1.2} \mathrm{O}_{4}$ nanocubes with an interconnected channel structure as high-performance anodes for lithium ion batteries. J Mater Chem A, 2015, 3: 2815-2824

46 Cai X, Gao W, Ma M, et al. A Prussian blue-based core-shell hollow-structured mesoporous nanoparticle as a smart theranostic agent with ultrahigh $\mathrm{pH}$-responsive longitudinal relaxivity. Adv Mater, 2015, 27: 6382-6389

47 Zakaria MB, Hu M, Hayashi $\mathrm{N}$, et al. Thermal conversion of hollow Prussian blue nanoparticles into nanoporous iron oxides with crystallized hematite phase. Eur J Inorg Chem, 2014, 2014: 1137-1141

48 Zhu T, Chang S, Song YF, et al. PVP-encapsulated $\mathrm{CoFe}_{2} \mathrm{O}_{4} / \mathrm{rGO}$ composites with controllable electromagnetic wave absorption performance. Chem Eng J, 2019, 373: 755-766

49 Song Y, He L, Zhang X, et al. Highly efficient electromagnetic wave absorbing metal-free and carbon-rich ceramics derived from hyperbranched polycarbosilazanes. J Phys Chem C, 2017, 121: 2477424785

50 Liu S, Liu J, Dong X. Electromagnetic Shielding and Absorbing Materials. 2nd ed. Beijing: Chemical Industry Press, 2014, 418

51 Zhao Y, Liu L, Jiang K, et al. Distinctly enhanced permeability and excellent microwave absorption of expanded graphite $/ \mathrm{Fe}_{3} \mathrm{O}_{4}$ nanoring composites. RSC Adv, 2017, 7: 11561-11567

52 Luo C, Jiao T, Gu J, et al. Graphene shield by SiBCN ceramic: A promising high-temperature electromagnetic wave-absorbing material with oxidation resistance. ACS Appl Mater Interfaces, 2018, 10: 39307-39318

53 Luo C, Tang Y, Jiao T, et al. High-temperature stable and metalfree electromagnetic wave-absorbing SiBCN ceramics derived from carbon-rich hyperbranched polyborosilazanes. ACS Appl Mater Interfaces, 2018, 10: 28051-28061

54 Li J, Miao P, Chen KJ, et al. Highly effective electromagnetic wave absorbing prismatic $\mathrm{Co} / \mathrm{C}$ nanocomposites derived from cubic metal-organic framework. Compos Part B-Eng, 2020, 182: 107613

Acknowledgements This work was financially supported by the $\mathrm{Na}$ tional Natural Science Foundation of China (21875190), Polymer Electromagnetic Functional Materials Innovation Team of Shaanxi Sanqin Scholars, the Natural Science Basic Research Plan in Shaanxi Province of Distinguished Young Scholar (2018JC-008), and China Postdoctoral Science Foundation (2018M643724).

Author contributions Kong J and Chen KJ designed the experiments. Kong J led the project. Miao P and Chen J conducted and performed the experiments. Kong J, Chen KJ and Tang Y analyzed the data. Miao P, Kong $\mathrm{J}$ and Chen KJ wrote the manuscript. All authors contributed to the general discussion.

Conflict of interest The authors declare that they have no conflict of interest.

Supplementary information Supporting data are available in the online version of the paper. 


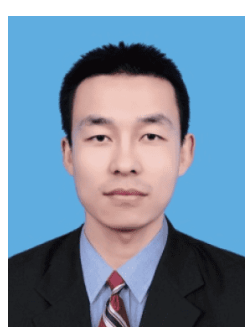

Peng Miao received his master degree in materials physics and chemistry from Chang'an University in 2014 . He is currently a $\mathrm{PhD}$ candidate majored in chemistry under the supervision of Prof. Jie Kong at Northwestern Polytechnical University. His research interest mainly focuses on the synthesis of metal-organic frameworks and their applications in electromagnetic absorption and shielding.

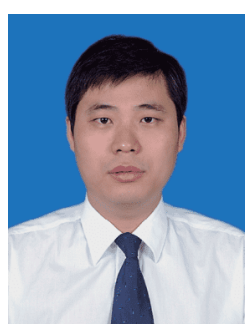

Jie Kong received his $\mathrm{PhD}$ degree from Northwestern Polytechnical University in 2004. He then went to The Hong Kong Polytechnic University as a postdoctoral fellow and the University of Bayreuth as an Alexander von Humboldt research fellow. In 2011, he joined the School of Science at Northwestern Polytechnical University as a full professor. His research interests include hyperbranched polymers, metal-organic frameworks, ceramic precursors and electromagnetic absorbing/transmitting materials.

\section{金属有机框架衍生高效宽频电磁波吸收剂拓扑结 构调控研究}

苗鹏, 陈建新, 唐玉生, 陈凯杰, 孔杰*

摘要 装备隐身和电子器件电磁防护的发展对高带宽、强吸收先 进电磁波吸收材料具有迫切需求, 采用金属有机骨架(MOF)衍生有 序结构金属/碳杂化材料是重要制备方法. 本文采用具有可控拓扑 结构和微相组成的普鲁士蓝衍生物作为前驱体, 通过热解工艺制 备空心、实心的笼状、盒状等拓扑结构 $\mathrm{Fe} / \mathrm{Co} / \mathrm{C}$ 和 $\mathrm{Fe} / \mathrm{Mn} / \mathrm{C}$ 纳米复 合电磁波吸收剂. 实心 $\mathrm{Fe} / \mathrm{Co} / \mathrm{C}$ 和空心 $\mathrm{Fe} / \mathrm{Mn} / \mathrm{C}$ 均表现出强电磁吸 收和高吸收带宽, 电磁波反射系数最低为 $-54.6 \mathrm{~dB}$, 其中 $900^{\circ} \mathrm{C}$ 热解 制备的实心盒状 $\mathrm{Fe} / \mathrm{Co} / \mathrm{C}$ 吸收剂在厚度 $2.5 \mathrm{~mm}$ 时有效吸收带宽高 达 $8.8 \mathrm{GHz}$ ，刷新了该类材料的性能上限. 通过控制MOF拓扑结构 来设计和调控金属/碳杂化吸波剂对于新型电磁波吸收材料的发展 及其在装备隐身领域的应用具有重要意义. 